\title{
On line cerebral blood flow velocity and blood pressure measurement in neonates: a new method
}

\author{
A C Fenton, D H Evans, M I Levene
}

\begin{abstract}
To test the hypothesis that impairment of cerebral perfusion and cerebrovascular autoregulation play a part in the pathogenesis of neurological injury in the critically sick neonate, we tested in 33 infants a small, lightweight probe and cable that are attached to the infant's skin to record cerebral blood flow velocity from the middle cerebral artery over a period of hours. This considerably reduced the amount of handling of the infant compared with conventional assessment. Captured data were analysed and displayed graphically at the cotside. The system is applicable for use on infants over a wide range of gestational ages and may give information on the complex haemodynamic changes occurring in the cerebral circulation.
\end{abstract}

Advances in neonatal intensive care, most notably in aspects of ventilatory support, have led to the prolonged survival of many extremely preterm, low birthweight infants as well as infants born at full term with various potentially life threatening disorders. A minority of surviving infants subsequently develop cerebral palsy or other neurodevelopmental problems. These may be the cause of long term financial and emotional strain on both their families and on the medical services.

The immature neonatal brain is extremely vulnerable to ischaemic and haemorrhagic injury, and the hypothesis is that disturbances of cerebral perfusion play a part in the pathogenesis of such injury. ${ }^{1}$ This has prompted several studies that have attempted to relate circulatory indices to the subsequent development of cerebral injury to identify the major aetiological factors. ${ }^{2} 3$ Despite these, the train of pathological events are not completely understood.

Changes in cerebral blood flow velocity correlate well with actual changes in cerebral blood flow. ${ }^{4}$ Studies of cerebral blood flow velocity with conventional duplex scanners are limited to being done once or twice a day to minimise the handling that critically ill premature infants tolerate so badly.

This regimen has several potential drawbacks. First, there may be failure to detect both acute changes in cerebral blood flow velocity that occur between studies (for example in association with pneumothorax or haemorrhage) and more chronic changes occurring over a period of several hours. In addition, the handling that is inevitable in such studies, even if minimal, may affect the validity of the results obtained. For this reason we have assembled a system that enables us to record, analyse, and display cerebral blood flow velocity from the middle cerebral artery, as well as measurements of blood pressure, at preset or manually determined intervals with a small button transducer that is fixed to the infant's skin, thus obviating the need for repeated handling.

\section{Methods}

HARDWARE

The system is shown schematically in fig 1 . The pressure front end is attached to an indwelling arterial catheter, allowing measurement of blood pressure as previously described. ${ }^{5}$ The Doppler unit is a Doptek $4 \mathrm{MHz}$ pulsed wave unit with a small button transducer (diameter $11 \mathrm{~mm}$, depth $7 \mathrm{~mm}$ ). The ultrasound intensity is below $50 \mathrm{~mW} / \mathrm{cm}^{2}$ spatial peak temporal average.

The use of pulsed wave ultrasound has two main advantages over continuous wave in this particular setting. First, it requires only a single, fine coaxial cable between the Doppler unit and transducer, which reduces cable drag on the transducer and makes fixation easier. Secondly, it allows the signals from the ipsilateral and contralateral middle cerebral arteries to be distinguished, even when reverse flow is present.

The remainder of the system comprises a spectrum analyser and microcomputer. The entire assembly occupies a small trolley that may be left at the cotside and does not interfere with routine nursing and medical care. The small size of the transducer is most acceptable to parents.

\section{FIXATION}

Positioning and fixing the transducer is critical because it must collect data over several hours. The Doppler unit is activated and the transducer moved over the temporal bone until the optimal signal is obtained from the ipsilateral middle cerebral atery. The transducer is then fixed in position using collodion. After fixation, the sample volume depth and length may be adjusted further to optimise the signal. Positioning and fixing the transducer normally takes three or four minutes.

\section{ANALYSIS}

Once set up the software activates the Doppler unit at preset intervals. In addition there is a manual over-ride facility, allowing the system to 


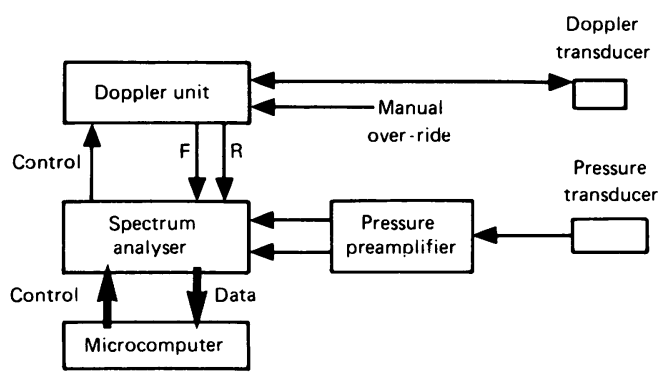

Figure 1 Schematic layout of on line system. $F=$ forward, $R=$ reverse.

be activated to capture changes in cerebral blood flow velocity and blood pressure associated with changes in the infant's clinical condition.

After activation, 12 seconds of blood pressure and spectrum analysed Doppler data are acquired by the microcomputer. The Doppler unit is then switched off. A maximum frequency envelope is derived from the Doppler data, ${ }^{6}$ and after the data are split into individual cardiac cycles, various measurementsincluding mean velocity (and its coefficient of variation), received Doppler power, and resistance area produced ( $\mathrm{RAP}=$ mean blood pressure/mean blood flow velocity), an index of cerebrosvascular resistance-are calculated. ${ }^{7}$ The results are written to disk and displayed graphically at the cotside.

\section{Results}

We have used the system on 33 infants. Details of the infants studied are given in the table. Informed consent was obtained before each study, and parents were encouraged to be present for all or part of the study.

The gestational age (24-41 weeks) and weight range $(750-3510 \mathrm{~g})$ of the group studied is representative of the population seen in most neonatal intensive care units. The average length of study was 4.5 hours (range 2-8), and in most cases ( 29 of 33 studies) it was decided electively to discontinue the study. In four cases the infant's own movements or the nurses turning the baby caused appreciable degradation in signal quality to the extent that further recordings were not possible without reapplication of the transducer. The longest period of continuous monitoring without reapplying the transducer was 48 hours.

Studies were planned to begin immediately after nursing care had been given to minimise periods of handling. The study was discontinued if a change in position at the next care resulted in the infant's head lying on the Doppler transducer, which we feared might

Details of infants studied

\begin{tabular}{lcc}
\hline Gestation (weeks) & No of infants & Weight range $(\mathrm{g})$ \\
\hline$<30$ & 15 & $750-1470$ \\
$30-34$ & 11 & $1230-1710$ \\
$>34$ & 7 & $1920-3510$ \\
\hline
\end{tabular}

damage the sensitive skin. Alternatively the transducer could be reapplied to insonate the contralateral middle cerebral artery.

The main problem encountered so far has been to maintain the transducer alignment and hence the quality of the Doppler signal for prolonged periods. The system works best in relatively inactive or therapeutically paralysed infants who are receiving minimal nursing and medical handling. This group of infants tends to be the most critically ill and therefore most at risk of developing neurological complications. It is envisaged that in practice the transducer should be checked every four hours and reapplied if necesseary in much the same way that transcutaneous gas monitors are used. In this way monitoring can be continued for days.

We have used the system to study normal variations in cerebral blood flow velocity and blood pressure and have also recorded the changes that occur in response to drugs and to ventilatory adjustment.

A typical trace from an infant of 29 weeks' gestation being ventilated for respiratory distress syndrome is shown in fig 2. Several features on the traces are worthy of note. Inadequate flushing of the indwelling arterial line resulted in damping of the blood pressure trace (point $a$, upper trace). Any activity on the part of the infant or any handling resulted in a change in cerebral blood flow velocity, in this case an increase (point $b$, middle trace). This highlights the inadequacies of intermittent sampling of cerebral blood flow velocity with conventional equipment.

Carbon dioxide is a potent vasodilator, and the cerebral vasculature is extremely sensitive to fluctuations in arterial carbon dioxide tension $\left(\mathrm{PaCO}_{2}\right)$, that are mediated by changes in the

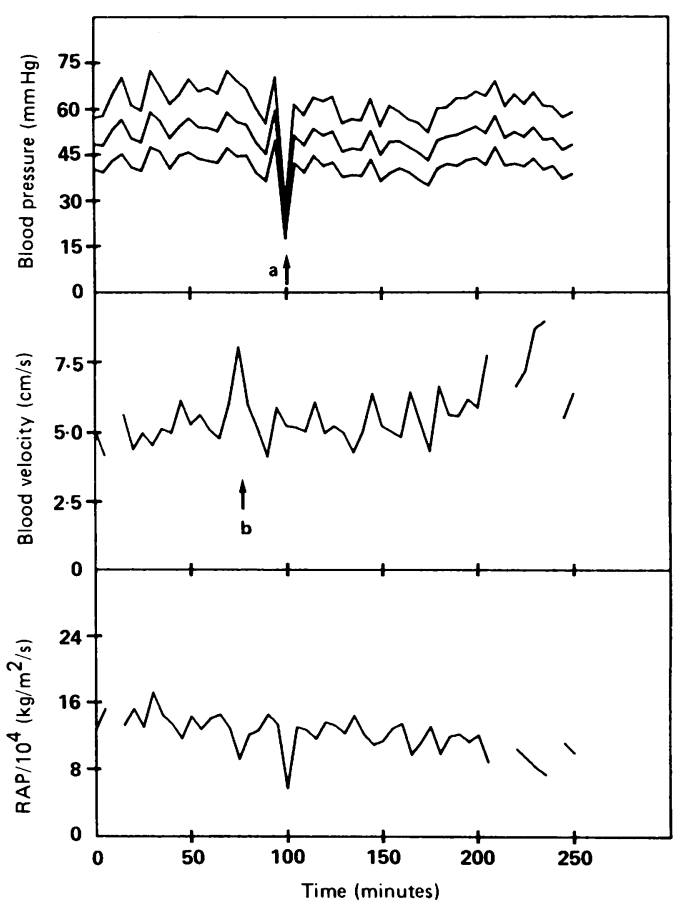

Figure 2 Normal fluctuations in cerebral blood flow velocity. $R A P=$ resistance area produced (mean blood pressure/mean blood flow velocity). 
$\mathrm{pH}$ of the cerebral interstitial fluid. A reduction in the inspiratory pressure of an infant being ventilated for respiratory distress syndrome resulted in an immediate rise in cerebral blood flow velocity (fig 3, point c). Repeat arterial blood gas sampling (point $d$ ) confirmed the rapid rise in $\mathrm{PaCO}_{2}$, and returning the ventilator pressure to its original setting restored the carbon dioxide tension and cerebral blood flow velocity

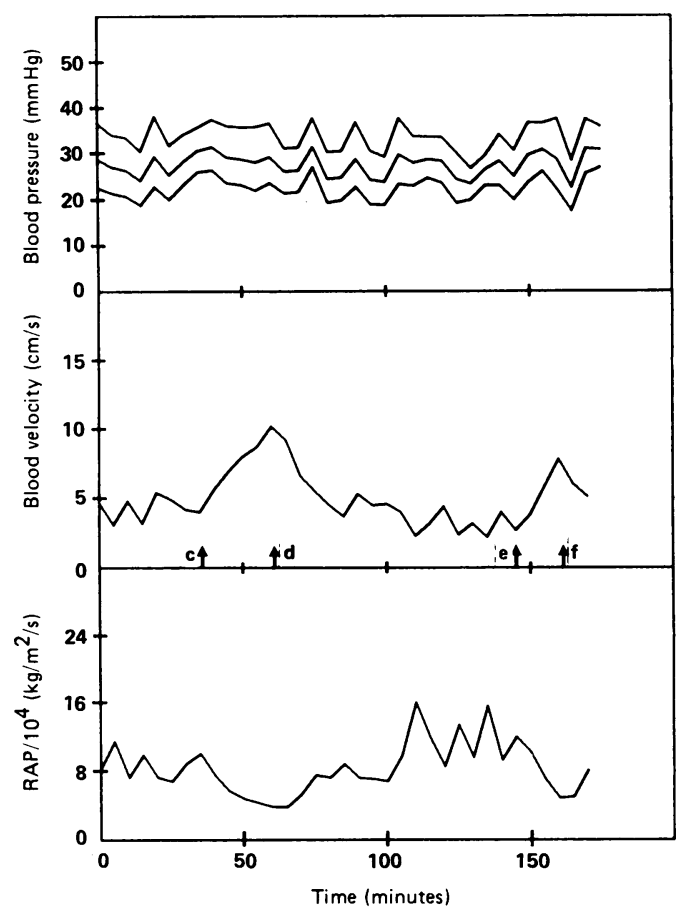

Figure 3 Effect of carbon dioxide on cerebral blood flow velocity. $R A P=$ resistance area produced (mean blood pressure/mean blood flow velocity).

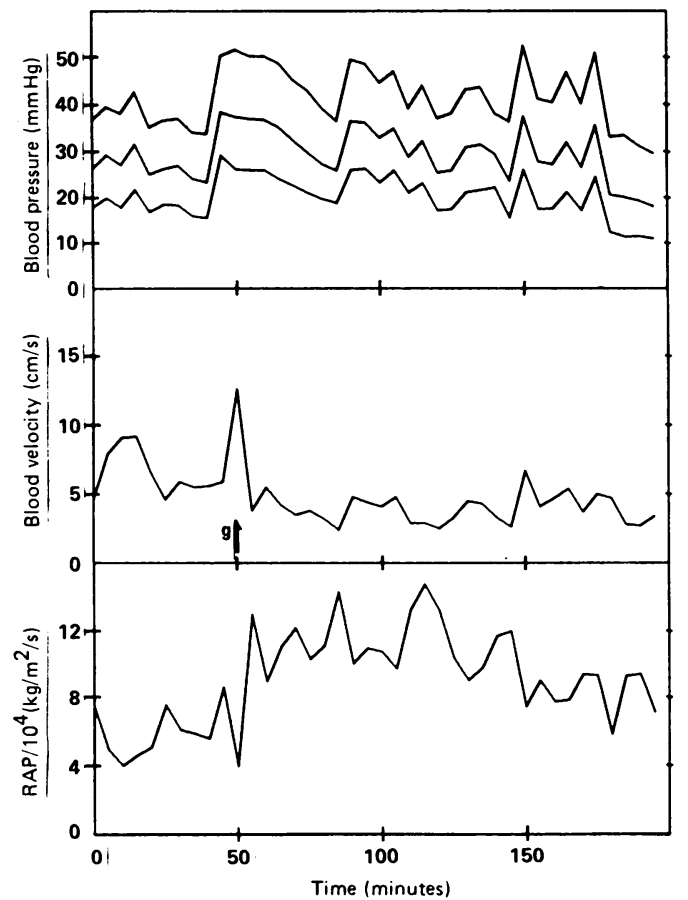

Figure 4 Effect of indomethacin on cerebral blood flow velocity. $R A P=$ resistance area produced (mean blood pressure/mean blood flow velocity). to their previous levels. A similar effect may be obtained by inserting a dead space into the ventilator circuit (point $e$ ), which causes a rise in $\mathrm{PaCO}_{2}$, and then removing it (point $f$ ).

Several drugs that may have profound effects on the circulation are used in neonatal intensive care. For example, indomethacin is used in the medical management of patent ductus arteriosus. Giving indomethacin (fig 4, point $g$ ) resulted in a sustained fall in cerebral blood flow velocity and a sustained rise in resistance area product, which presumably indicates vasoconstriction. ${ }^{8}$ The rise in cerebral blood flow velocity immediately before the drug was given was caused by handling the infant.

\section{Discussion}

We have observed no local adverse effects from fixation of the transducer and believe it to be safe in terms of power output. ${ }^{9}$ The system is simple, can be rapidly set up, and may be used to collect data in two ways. First, it allows the study of both normal and pathological variations in cerebral blood flow velocity in critically ill neonates over periods of several hours and relates these to changes in blood pressure, while adhering to the principle of minimal handling. More continuous measurement of changes in cerebral blood flow velocity is more likely to detect important changes that correlate with the development of ischaemic or haemorrhagic cerebral events.

Secondly, it may be used to monitor rapid changes in cerebral haemodynamics resulting from ventilatory or pharmacological manipulation, or from clinical deterioration. During the course of evaluating this equipment it has become apparent that wide fluctuations in cerebral blood flow velocity may occur as a result of handling the infants. In non-paralysed infants this may be caused, in part, by breathing asynchronously with the ventilator. Perlman et al have suggested that fluctuating cerebral blood flow velocity causes intraventricular haemorrhage, ${ }^{10}$ but we suspect that merely placing the ultrasound probe on the infant's head is enough to cause these abnormal patterns. It is not yet clear whether fluctuations in cerebral blood flow velocity as described by Perlman et al, if present in infants who are not disturbed, are as important as those induced by handling. Our equipment obviates the need for repeated handling, allows more detailed study of the ill infant's spontaneous interaction with the ventilator, and will help us to determine the answer to this important question.

This system will also allow the investigator to assess the effect of spontaneous changes in blood pressure on cerebral blood flow velocity in an attempt to provide further evidence for the failure of cerebrovascular autoregulation in the pathogenesis of neurological injury in the critically ill newborn infant. It may help us to formulate and monitor management regimens aimed at reducing the incidence of such injury.

We thank Mr M Teague and Mr C Cornhill of Doptek (UK) Ltd for the loan of the equipment and production of the transducer. ACF is supported by the Spastics Society. 
1 Pape KF, Wigglesworth JS. Haemorrhage, ischaemia and the perinatal brain. Clinics in developmental medicine. No 69/70. London: Spastics International Medical Publications, 1979. 2 Weindling AM, Wilkinson AR, Cook J, Calvert SA, Fok T-F, Rochefort MJ. Perinatal events which precede periventricular haemorrhage and leukomalacia in the newborn. Br f Obstet Gynaecol 1985;92:1218-23.

3 Watkins A, West C, Cooke RWI. Blood pressure and cerebral injury in sick very low birthweight infants. Arch Dis Child 1987;62:648-9.

4 Hansen NB, Stonestreet BS, Rosenkrantz TS, Oh W. Validity of Doppler measurements of anterior cerebral artery blood flow: correlation with brain blood flow in piglets. Pediatrics 1983;72:526-31.

5 Evans DH, Lark GM, Archer LNJ, Levene MI. The continuous measurement of intra-arterial pressure in the neonate: method and accuracy. Clin Phys Physiol Meas 1986;7:179-84.

6 Schwindlein FS, Smith MJ, Evans DH. Spectral analysis of
Doppler signals and computation of the normalised first moment in real time using a digitial signal processor. Med Biol Eng Comput 1988;26:228-32.

7 Evans DH, Levene MI, Shortland DB, Archer LNJ. Resistance index, blood flow velocity, and resistance area product in the cerebral arteries of very low birth weight infants during the first week of life. Ultrasound Med Biol 1988;14:103-10.

8 Levene MI, Shortland DB, Gibson N, Evans DH. Carbon dioxide reactivity of the cerebral circulation in extremely
premature infants: effects of postnatal age and indomethacin. Pediatr Res 1988;24:175-9.

9 American Institute for Ultrasound Medicine. Safety considerations for diagnostic ultrasound. Bethesda: American Institute of Ultrasound in Medicine, 1984.

10 Perlman JM, McMenamin JB, Volpe JJ. Fluctuating cerebral blood flow velocity in respiratory distress syndromerelation to the development of intraventricular haemorrhage. relation to the development of in 